\title{
Un pas de plus vers la pathogénie de la maladie d'Alzheimer? Interactions entre préséniline-1 et $\beta$-caténine
}

La découverte de mutations de la préséniline-1 dans les maladies d'Alzheimer familiales a permis d'ouvrir des pistes de recherche sur la pathogénie de la maladie $\left(\mathrm{m} / \mathrm{s} 1995, n^{\circ} 11\right.$, p. 1610). Il a vite été montré que la préséniline-1 mutante favorisait l'accumulation du peptide amyloïdogène $A \beta 42$, un des principaux constituants de la plaque sénile $(\mathrm{m} / \mathrm{s} 1997$, $\left.n^{\circ} 1, p .106\right)$. Par ailleurs, le gain de fonction de la préséniline-1 mutée la fait interagir plus fortement avec la kinase GSK-3 $\beta$ et la protéine tau: cela pourrait favoriser l'hyperphosphorylation de la protéine tau et la formation des filaments en hélice neurofibrillaires de protéine tau caractéristiques des lésions neuronales de la maladie d'Alzheimer [1, 2]. Aujourd'hui c'est une étude des voies conduisant à une augmentation de l'apoptose neuronale dans la maladie qui est rapportée [3]. Récemment est apparue l'information que préséniline-1 et $\beta$ caténine s'associaient dans la cellule [4] ; la préséniline est une protéine à huit passages transmembranaires, localisée essentiellement dans les membranes du réticulum endoplasmique et de l'appareil de Golgi, clivée en grande partie en deux fragments amino- et carboxy-terminaux (NTF et CTF) qui forment avec la $\beta$ caténine un complexe de $250 \mathrm{kDa}$; la $\beta$-caténine, pour sa part, est une protéine importante dans la transmission intracellulaire de nombreux signaux et la régulation de sa stabilité est un point crucial de la biologie cellulaire. Elle a deux localisations majoritaires, sous la surface cellulaire, liée à la E-cadhérine, et dans le cytoplasme [3]. Comme cela a été plusieurs fois discuté dans $\mathrm{m} / \mathrm{s}$, la $\beta$ caténine joue un double rôle: dans l'adhérence intercellulaire, comme un des constituants des jonctions serrées entre cellules épithéliales, et comme un médiateur de la transmission du signal Wingless/Wnt [5-7]. Lorsque la kinase GSK-3 $\beta$ est inhibée sous l'effet du signal Wnt, la $\beta$-caténine est déphosphorylée, stabilisée et forme un complexe d'activation transcriptionnelle avec des facteurs de la famille LEF/Tcf. Préséniline-1 et $\beta$-caténine sont colocalisées dans le complexe de Golgi [3] et on peut imaginer que la préséniline y séquestre la $\beta$-caténine. Cette interaction stabiliserait la $\beta$-caténine, et c'est la perte de cette action de la préséniline mutée qui entraînerait la déstabilisation de la caténine: non seulement elle est en moindre abondance dans le cortex cérébral de souris transgéniques exprimant une préséniline mutée et de malades ayant une maladie d'Alzheimer familiale $(-46 \%)$, mais on observe aussi la présence de toute une série de ses produits de dégradation. Des études in vitro, sur des cultures de fibroblastes provenant de souris au gène de la préséniline-1 invalidé, confirment cette observation, insistant sur la perte de cette fonction due à la mutation de PS1 ou à son absence. La diminution de la concentration de $\beta$-caténine a-telle une responsabilité dans la dégénérescence neuronale ? L'étude a été effectuée sur des cultures primaires de neurones de l'hippocampe de rat, transfectées avec des vecteurs exprimant la $\beta$-caténine normale, ou une construction dominante-négative dont le produit inhibe la transmission du signal qui passe par la famille LEF de facteurs de transcription [3]. L'expression de la $\beta$-caténine anormale s'accompagne d'une augmentation importante de l'apoptose de ces neurones induite par la protéine fibrillaire $\beta$ amyloïde. La co-expression de la $\beta$ caténine normale supprime l'apoptose. Il faut bien souligner que l'apoptose observée dépend absolument de la présence du peptide $\beta$ amyloïde. L'interprétation des auteurs est que le complexe transcriptionnel actif constitué des facteurs LEF/Tcf liés à la $\beta$-caténine protègeraient de l'apoptose neuronale due à ce peptide.

Ces résultats sont en apparente contradiction avec le rôle décrit récemment pour la protéine Armadillo, équivalent drosophilien de la $\beta$ caténine, par l'équipe de Eric Wiechaus (Princeton, NJ, USA) : selon ces chercheurs, le complexe Armadillo-dTcf induirait une apoptose des neurones rétiniens [8]. Ce phénomène pourrait expliquer les lésions rétiniennes associées à certaines formes de polyposes coliques familiales avec mutation du gène $A P C$, réunies sous le nom d'hypertrophie congénitale de l'épithélium pigmentaire rétinien [8]. En effet, la perte de fonction d'APC aboutit à une stabilisation de la $\beta$-caténine et à une activation anormale du complexe $\mathrm{LEF} / \beta$-caténine.

$\mathrm{Au}$ total, tous ces résultats concourent pour suggérer que les présénilines interviennent dans le contrôle de phénomènes protéolytiques: (1) les présénilines sont les homologues du gène Sel-12 de C. elegans, nécessaire à la transmission du signal Notch [9]; la fonction de ce gène peut, en effet, être complémentée par PS1 [10]. Or, la fixation sur Notch de ses ligands provoque un cli- 
vage protéolytique de la région intracytoplasmique liée au facteur de transcription $\mathrm{Su}(\mathrm{H})$ (suppressor of hairless) [11]. (2) La préséniline 1 semble contrôler la dégradation de la $\beta$-caténine, et donc le signal Wnt. Cependant, on ne comprend pas encore bien si la sensibilisation des neurones à l'apoptose est un phénomène important dans la maladie d'Alzheimer, et si elle est facilitée ou plutôt inhibée par l'activation du complexe LEF/ $\beta$-caténine.
1. Takashima A, Murayama M, Murayama $\mathrm{O}$, et al. Presenilin 1 associates with glycogen synthase kinase-3B and its substrate tau. Proc Natl Acad Sci USA 1998; 95: 9637-41.

2. Tranchant C. Protéines TAU et maladies neurologiques. Med Sci 1997; 13: 989-97.

3. Zhang Z, Hartmann H, Do VM, et al. Destabilization of $\beta$-catenin by mutations in presenilin-1 potentiates neuronal apoptosis. Nature 1998; 395: 698-702 4. Yu G, Chen F, Levesque G, et al. The presinilin 1 protein is a component of a high molecular weight intracellular complex that contains $\beta$-catenin. J Biol Chem 1998; 273: 16470-5.

5. Bornens M, Camonis J, Goud B, Thiéry J, Louvard D. Le nouvel âge de la cellule. Med Sci 1996; 12: 50-66.

6. Romagnolo B. APC: de nouveaux partenaires, de nouveaux indices... Med Sci 1996; 12: 1109-10.

7. Romagnolo B. APC, $\beta$ caténine et cancer: les E.B. diaboliques. Med Sci 1997; 13: 872-3.
8. Ahmed Y, Hayashi S, Levine A, Wiechaus E. Regulation of Armadillo by a Drosophila APC inhibits neuronal apoptosis during retinal development. Cell 1998; 93: 1171-82.

9. Levitan D, Greenwald I. Facilitation of lin-12mediated signalling by sel-12, a Caenorhabditis elegans S182 Alzheimer's disease gene. Nature 1995; 377: $351-4$

10. Baumeister R, Leimer U, Zweckbronner I, Jakubek C, Grunberg J, Haass C. Human presenilin-1, but not familial Alzheimer's disease (FAD) mutants, facilitate Caenorhabditis elegans Notch signalling independently of proteolytic processing. Genes Funct 1997; 1 : 149-59.

11. Schweisguth F, Israël A. Signalisation intercellulaire par le récepteur Notch: conservation de la drosophile aux mammifères. Med Sci 1996; 12: 155-63.

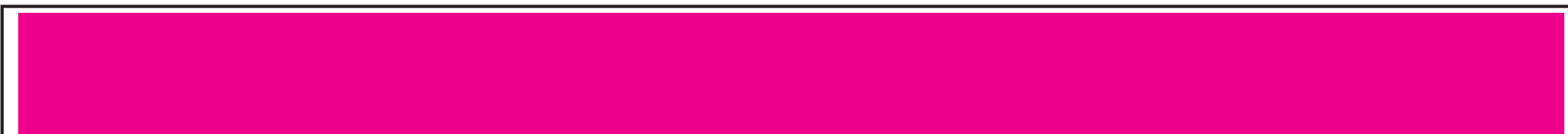

